\title{
Delirium in older people
}

\author{
John Young, ${ }^{1}$ Sharon K Inouye ${ }^{2}$
}

${ }^{1}$ Academic Unit of Elderly Care and Rehabilitation, University of Leeds and Bradford Teaching Hospitals NHS Foundation Trust

${ }^{2}$ Department of Medicine, Beth Israel Deaconess Medical Center, Harvard Medical School, and

Aging Brain Center, Institute for Aging Research, Hebrew Senior Life, Boston, MA, USA

\section{Correspondence to: Professor}

J Young, Academic Unit of Elderly Care and Rehabilitation, St Luke's Hospital, Bradford BD5 ONA

John.young@bradfordhospitals. nhs.uk

BMJ 2007;334:842-6

doi: 10.1136/bmj.39169.706574.AD
Few ill health situations are more degrading to people of any age than loss of reasoning, faculties, and personhood. These are the unpleasant consequences of delirium - a common condition affecting ill older people, particularly those with some degree of dementia. It is characterised by recent onset of fluctuating inattention and confusion, linked to one or more triggering factors.

Delirium is a major burden to healthcare services and has been largely ignored by health service planners and practitioners. ${ }^{1}$ Moreover, healthcare systems and services often unintentionally stimulate or substantially aggravate the development of delirium in older people. ${ }^{2}$ This might be understandable if delirium was unavoidable or untreatable, but the existing evidence base for delirium is sufficiently robust for prevention or attenuation of the condition to be a realistic proposition. There is a pressing need to take this action because the outcomes for delirium are poor: it contributes to substantial morbidity and mortality, causes considerable distress to patients and families, and is expensive - an estimated additional \$2500 ( $£ 1275$; $€ 1875$ ) per patient (a $\$ 6.9 \mathrm{bn}$ annual expenditure for Medicare in 2004). ${ }^{3}$

\section{Sources and selection criteria}

We searched Medline and the Cochrane Library from 1996 to 2006 . We drew additional material from our personal libraries of delirium references, focusing particularly on systematic reviews.

\section{How common is delirium?}

Delirium is an important problem for all clinical services providing care for older people, particularly emergency departments; general medical, elderly care, surgical, and oncology wards; intensive care units; and, in the community, residential and nursing homes. Delirium is the most common complication of hospital admission for older people. ${ }^{\mathrm{w} 1}$ It develops in up to a half of older patients postoperatively; especially after hip fracture and vascular surgery. ${ }^{\mathrm{w} 2 \mathrm{w} 3} \mathrm{~A}$ systematic review that identified 42 studies of occurrence and outcomes of delirium in medical inpatients found that the occurrence of delirium varied between $11 \%$ and $42 \% .{ }^{4}$ These rates are likely to be underestimates, resulting from bias in study recruitment, which favours people without dementia (therefore with a lower risk of delirium).$^{5}$

\section{What are the consequences of delirium?}

Estimates for outcomes for delirium are influenced by elements of study design such as case ascertainment, confounder adjustment, and adequacy of follow-up. ${ }^{\mathrm{w} 4}$ A well conducted, prospective comparison study of medical patients with delirium reported a twofold increase in discharge mortality, an average increase of eight days in the length of stay in hospital, and worse physical and cognitive recovery at six and 12 months with increased time in institutional care. ${ }^{6}$ Good evidence also exists that symptoms of delirium persist in about a third of patients ${ }^{4}$ and that these patients have a worse prognosis. ${ }^{7}$ After recovery from delirium, patients can experience recollections of the event that they find unpleasant and disturbing. ${ }^{\text {w5 }}$

\section{What are the clinical features of delirium?}

The cardinal features of delirium are recent onset of fluctuating awareness, impairment of memory and attention, and disorganised thinking (box 1). ${ }^{8}$ Additional features may include hallucinations and disturbance of the sleep-wake cycle. There are three clinical subtypes of delirium: hyperactive, hypoactive, and mixed (boxes 2 and 3). Hypoactive delirium is less likely to be recognised despite patients having a more severe illness. ${ }^{9}$ The term subsyndromal delirium has been suggested for patients who have an incomplete form of delirium. ${ }^{\mathrm{w} 6}$

\section{How is delirium diagnosed?}

The diagnosis of delirium rests solely on clinical skills; no diagnostic test exists. This may partly explain why it is undiagnosed in over a half of patients with the condition. $^{\mathrm{w} 7 \mathrm{w} 8}$ Delayed or missed diagnosis is an important issue - non-detection of delirium in emergency departments is associated with a sevenfold hazard for increased mortality. ${ }^{\mathrm{w} 9}$ Healthcare professionals often describe a patient with possible delirium as "confused elderly patient," but this fails to distinguish between delirium and dementia. Obtaining the history of the clinical course of any cognitive changes from a family member or carer is key to recognising delirium. A diagnosis of delirium will stimulate the appropriate, probing clinical questions: what has happened to the patient; what are the underlying factors; how can they be resolved; and could the delirium have been prevented? 
Box 1 | Criteria for delirium, according to the Diagnostic and Statistical Manual of Mental Disorders (DSM IV) ${ }^{8}$

- Disturbance of consciousness with reduced ability to focus, sustain, or shift attention

- Changed cognition or the development of a perceptual disturbance

- Disturbance develops in a short period of time and fluctuates over the course of the day

- History, physical examination, and laboratory findings show that delirium can be a physiological consequence of general condition; caused by intoxication; caused by medication; and caused by more than one aetiology

A core feature of delirium is its impact on cognitive function. Guidelines therefore recommend that all clinical encounters with sick older people should routinely include assessment of cognition. ${ }^{10}{ }^{11}$ The minimental state examination (30 questions) ${ }^{\mathrm{w} 10}$ and the abbreviated mental test (10 questions) ${ }^{\text {w11 }}$ are widely used in routine care, but full completion is often difficult in an acute setting in patients who are ill or in pain. The cognitive impairment test (six questions) ${ }^{\text {w12 }}$ and the four item abbreviated mental test score ${ }^{\mathrm{w} 13}$ correlate well with the mini-mental state examination and are simple to administer. Alternatively, quick screening instruments for inattention include the digit span test and listing days of the week backwards. Sequential cognitive assessments-for example, using the minimental state examination daily-can successfully monitor development and resolution of delirium. ${ }^{12}$

Several diagnostic instruments have been developed for detecting delirium. US and UK guidelines recommend the confusion assessment method for routine use. ${ }^{10113}$ The shorter form has four questions (box $4)$; it performs as well as the more complex delirium rating scale ${ }^{\mathrm{w} 14}$ and can be completed in less than five minutes. ${ }^{\text {w15 }}$ Appropriate training and use of a brief cognitive assessment instrument ${ }^{14}$ results in a sensitivity and specificity of over $90 \% .^{13}$

\section{The overlap between delirium and dementia}

Distinguishing delirium from dementia is a common clinical dilemma. There is a strong inter-relationship between delirium and dementia, both pathophysiologically ${ }^{\mathrm{w} 16}$ and clinically. ${ }^{15}$ Dementia is associated with an increased risk of developing delirium, and delirium is associated with increased risk of developing dementia, although it is unclear whether the delirium is unmasking previously unrecognised dementia or initiating a process of cognitive decline. ${ }^{15}$ It can be particularly difficult to distinguish delirium from Lewy body dementia because some features, such as hallucinations and symptom fluctuation, are common to both. The safest clinical approach is to consider that all older people presenting with confusion have delirium until proved otherwise (table 1). This implies completion of the cognitive assessments described above, obtaining an adequate history of the course of cognitive changes, and a thorough search for delirium recipitants.

\section{What are the causes of delirium?}

The pathophysiology of delirium is poorly understood; various concepts have been reviewed. ${ }^{\text {w17-w19 }}$ Good evidence suggests neurotransmitter disturbances, especially acetylcholine deficiency and dopamine excess. Less well supported mechanisms are illness related stress with overactivity of the hypothalamic-pituitary-adrenal axis, and the effects of increased cytokine production on cerebral function. A recent finding has been reduced activity of plasma esterases in delirium $^{\text {w20}}$; these are important drug metabolising enzymes and may partly explain why drugs are common precipitants for delirium. A unifying approach is to regard delirium as a clinical syndrome resulting from an interconnection of several pathological mechanisms. ${ }^{\mathrm{w} 17 \mathrm{w} 19}$

The concept of patient vulnerability (risk factors) in relation to stressor events (precipitants that trigger an episode of delirium) has proved a practical approach to understanding delirium. ${ }^{16}$ In most older patients, several precipitants may exist. The precipitants do not alone cause delirium; they interact with the underlying risk factors. Thus, a major insult, such as a serious infection, is required to trigger delirium in a previously fit person, but even a minor perturbation (such as a change in medication) can result in delirium in a person with many risk factors. Older people with multiple chronic diseases are therefore especially prone to delirium.

In older people the precipitants may present in occult or atypical fashion, confounding even experienced clinicians. Thus, a careful search for occult infection, myocardial infarction, metabolic disorders, or respiratory failure may be necessary.

Systematic reviews have assessed studies investigating risk factors in medical and surgical patients. ${ }^{\text {w21-w23 }}$

\section{Box 2 | Hyperactive delirium}

\section{Scenario}

An elderly woman has been admitted to your ward having been found lying on the floor by the home care staff. She seems to be talking to herself, sometimes loudly, but it is hard to understand what she is saying. She has a rather anxious demeanour and repeatedly pulls at her bedclothes. She argues with the nursing staff and has angrily refused to have a blood sample taken.

\section{Features}

In hyperactive delirium a patient has heightened arousal and can be very sensitive to his or her immediate surroundings, such that they can be verbally and physically threatening and aggressive. They can be restless, sometimes pulling repeatedly at clothing (called carphologia), and wandering is a common feature. 
Box 3 Hypoactive delirium

\section{Scenario}

An elderly man is recovering from a heart attack and seems to want to lie in bed. He is polite when approached, but sleepy, and he doesn't seem interested in eating or drinking. Sometimes his speech is incoherent and he doesn't seem to follow what is said to him.

\section{Features}

The clinical clues for delirium in this patient are much less obvious than in a patient with hyperactive delirium (box 2), although he displays all the major features of delirium. Hypoactive delirium is the more common of the two subtypes, but careful bedside observation skills are required to detect its more subtle features. This is one important reason why delirium is easily overlooked.

The reviews are limited by variation in quality and heterogeneity of the constituent studies. The commonly encountered risk factors and precipitants for delirium are listed in box 5 . Environmental risk factors for delirium include moves within the hospital, absence of a clock or watch, absence of reading glasses, presence of a family member, and use of restraints (physical or drugs). ${ }^{2}$

Understanding the risk factors for delirium provides an important opportunity to identify patients at high risk of developing the condition using predictive models ${ }^{16}$ w24-w26 and to target these patients for preventive interventions. Pre-existing dementia is common to all the predictive models and is the dominant risk factor associated with development of delirium.

\section{Drugs and delirium}

Drugs are an important risk factor and precipitant for delirium in older people: medications may be the sole precipitant for $12 \%-39 \%$ of cases of delirium. ${ }^{17}$ The
Box $4 \mid$ Confusion assessment method ${ }^{\star 1314}$

The diagnosis of delirium requires a present or abnormal rating for criteria 1 and 2 plus either 3 or 4.

\section{Acute onset and fluctuating course}

Is there evidence of an acute change in mental status from the patient's baseline? Did this behaviour fluctuate during the past day-that is, tend to come and go or increase and decrease in severity? (Usually requires information from a family member or carers)

\section{Inattention}

Does the patient have difficulty focusing attention-for example, are they easily distracted or do they have difficulty keeping track of what is being said? (Inattention can be detected by the digit span test or asking for the days of the week to be recited backwards)

\section{Disorganised thinking}

Is the patient's speech disorganised or incoherent, such as rambling or irrelevant conversation, unclear or illogical flow of ideas, or unpredictable switching between subjects? (Disorganised thinking and sleepiness can also be detected during conversation with the patient)

\section{Altered level of consciousness}

Overall, would you rate this patient's level of consciousness as alert (normal), vigilant (hyperalert), lethargic (drowsy, easily aroused), stupor (difficult to arouse), or coma (cannot be roused)? All ratings except alert are scored as abnormal

*Brief formal cognitive testing (such as the mini-mental state examination) is recommended for scoring.

most common drugs associated with delirium are psychoactive agents such as benzodiazepines, narcotic analgesics such as morphine, and drugs with anticholinergic effects. Many commonly used drugs in

Table1 Distinguishing delirium and dementia

Patient 1 (dementia)

\section{Scenario}

A 78 year old woman is found by her neighbours confused and wandering in the street at night wearing her night clothes. In the emergency room she appears unkempt and dishevelled. She is alert, but disoriented in time and place and cannot recall her home address. She engages well with questions, but tends to shift the conversation to stories about her husband and children. She is admitted to hospital and wanders around the ward appearing lost and, when asked, says that she is looking for a bus stop to go home

An 89 year old woman living alone is brought to your clinic by her daughter. The daughter explains that dementia was diagnosed at the local memory clinic about five years ago. Her mother has gradually deteriorated but has been coping at home with home care support. Two days ago she became incontinent of urine (unusual for her) and more confused in her conversation with the home care team, who also found her rather sleepy

\section{Distinguishing features}

All the clinical observations suggest that she does not have delirium. She is alert (no clouding of consciousness), engages well in conversation (no attention impairment). Although she has apparent night-time restlessness (altered sleep-wake cycle), this is explained by her search for a bus stop. The critical clinical action needed is to contact an informant who knows her. How long has she been confused? If she has a dementia, her family, neighbours, community support staff, or primary care team will know when she was last well and the duration of her confusional state

As this patient has dementia, she will be at especially high risk of delirium episodes. Features of delirium can be seen in this patient: short history of deterioration, disorganised conversation with the home care staff (who will know her usual abilities well), and observed sleepiness. The recent onset of urinary incontinence suggests that the delirium precipitant may be a urinaryinfection 
older people have anticholinergic effects, ${ }^{18}$ although these effects may not be well known (table 2). Whenever possible, these drugs should be discontinued in patients who are at risk of or have developed delirium.

\section{Can delirium be prevented?}

Most evidence for delirium prevention that informs clinical practice is from non-randomised clinical studies (although a Cochrane review of randomised con-

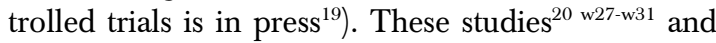
one randomised controlled trial ${ }^{21}$ collectively indicate that the most successful approach to delirium prevention is to attenuate modifiable risk factors in individual patients. This requires a complex intervention, and studies investigating such interventions in medical patients and those who have had hip fracture have reported significant reductions (of about a third) in incidence of delirium and/or reduced severity and duration of delirium. ${ }^{20} 21$ w27-w31

In contrast, the dissemination of good practice guidelines on delirium alone was only weakly effective $^{\text {w32 }}$ probably because the guidelines were not practical enough for the complexity of delirium so were not adhered to. Indeed, sustained adherence to the clinical protocol is a key factor for successful delirium prevention. ${ }^{22}$ The effective Yale delirium prevention model of care ${ }^{19}$ has been translated into routine practice as the hospital elder life program (HELP). ${ }^{23}$ This programme is now used in three countries and is associated with a reduction in the rates and costs of delirium. ${ }^{\text {w33 }}$

Effective strategies for delirium prevention include orienting communication, therapeutic activities, early mobilisation and walking, non-pharmacological approaches to sleep and anxiety, maintaining nutrition and hydration, adaptive equipment for vision and hearing impairment, and pain management. Hospitals have several inherent risks for the development of delirium $^{2}$ and early discharge to a home rehabilitation service was associated with a significantly reduced incidence of delirium. ${ }^{24}$

\section{How can delirium be managed?}

Immediate identification and treatment of precipitants (box 5), withdrawal of drugs (table 2), and supportive care (including management of hypoxia, hydration

\section{Box 5 Common risk factors and precipitants for delirium}

\section{Risk factors}

Old age (over 65 years), physical frailty, severe illness, multiple diseases, dementia, admission to hospital with infection or dehydration, visual impairment, deafness, polypharmacy, alcohol excess, renal impairment, malnutrition

Precipitants (more than one may be present) Lower respiratory tract infection, urinary infection/ catheters, constipation, electrolyte disturbance (dehydration, renal failure, hyponatraemia or hypernatraemia), drugs (especially those with anticholinergic activity or psychoactive drugs), alcohol withdrawal, pain, neurological disorder (stroke, epilepsy, subdural haematoma), hypoxia, sleep deprivation, surgery (such as fractured neck of femur), environmental (see text)

and nutrition, minimising the time spent lying in bed, and mobilisation) are the critical actions required. Three randomised controlled trials showed that input from a specialist, protocol based multidisciplinary team was no better than usual ward care..$^{25}$ w34 w35 Some hospitals have delirium units, but these have not been robustly evaluated. ${ }^{\text {w36 }}$ For patients with hyperactive delirium, physical restraints should be avoided because they tend to increase agitation and injury. ${ }^{\text {w37 }}$ Drug treatment should be reserved for patients who pose a risk to themselves or others, as the treatments may prolong or aggravate delirium in some cases.

A systematic review of psychotropic medication in delirium showed the paucity of reliable information available (four heterogeneous studies involving only 174 patients) but recommended low dose haloperidol as the best studied agent. ${ }^{\text {w38 }}$ The atypical antipsychotic drugs risperidone and olanzapine should be avoided in patients with dementia complicated by delirium because of the associated increased risk of stroke. $^{\text {w39 w40 }}$

\section{Conclusions}

Delirium is a major healthcare concern in countries with ageing populations. It is associated with poor

Table 2 Drugs with anticholinergic activity commonly used in older people

\begin{tabular}{ll} 
Type of drug & Example \\
Antihistamine & Hydroxyzine, diphenhydramine \\
\hline Antispasmodic & Alverine, hyoscyamine \\
\hline Tricyclic antidepressant & Amitriptyline \\
\hline Benzodiazepine & Lorazepam \\
\hline Analgesic & Codeine \\
\hline Antiarrhythmic & Digoxin \\
\hline Diuretic & Furosemide \\
\hline Antiparkinsonian & Orphenadrine, benzatropine (more often used in US than UK) \\
\hline Bladder stabiliser & Oxybutynin \\
\hline Brochodilator & Theophylline \\
\hline
\end{tabular}




\section{SUMMARY POINTS}

Delirium is a common presentation of acute illness in older people

The development of delirium is associated with adverse outcomes

Delirium is characterised by recent onset of fluctuating inattention and drowsiness linked to triggering factors

Routine cognitive assessment in unwell older people would improve detection rates

Good research evidence exists that, with better systems of routine care, delirium could be prevented in at least a third of patients

outcomes and is expensive. The existing research evidence suggests that delirium could be prevented in at least one third of cases, but new research is needed to better understand the causal mechanisms, including the relation of delirium to dementia. Unfortunately, health service planners and practitioners have yet to systematically tackle the potential for delirium prevention. Few national guidelines have been produced, ${ }^{\mathrm{w} 41}$ and delirium remains disproportionately ignored relative to its impact.

Contributors: JY planned the review, did the searches, and reviewed the literature. Both authors contributed to the writing. JY is the guarantor. Competing interests: None declared.

Provenance and peer review: Commissioned and externally peer reviewed.

\section{ADDITIONAL EDUCATIONAL RESOURCES}

\section{Resources for patients}

Mental Health Foundation (www.mentalhealth.org.uk/ information/mental-health-a-z/delirium/)

Hospital Elder Life Program (HELP) (www.

hospitalelderlifeprogram.org)

Resources for healthcare professionals

Brown TM, Boyle MF. ABC of psychological medicine: Delirium. BMJ 2002;325:644-7.

Clinical Effectiveness and Evaluation Unit. The prevention, diagnosis and management of delirium in older people. London: Royal College of Physicians of London, 2006. (www.rcplondon.ac.uk/pubs/books/ pdmd/index.asp)

Inouye S. Current concepts: delirium in older people. N Engl J Med 2006;354:1157-65.

Lindesay J, Rockwood K, Macdonald A, eds. Delirium in old age. Oxford: Oxford University Press, 2002.

Casey DA, DeFazio JV, Vansickle K, Lippmann SB. Delirium symposium. Postgraduate Medicine online, 1996 (www.postgradmed.com/issues/1996/07_96/ casey.htm)

Hospital Elder Life Program (HELP) (www. hospitalelderlifeprogram.org)

Practice guideline for the treatment of patients with delirium. American Psychiatric Association, 1999 (www.psych.org/psych_pract/treatg/pg/ Practice\%20Guidelines8904/Delirium.pdf)
1 Inouye SK, Schlesinger MJ, Lydon TJ. Delirium: a symptom of how hospital care is failing older persons and a window to improve quality of hospital care. Am J Med 1999; 106:565-73.

2 McCusker J, Cole M, Abrahamowicz M, Han L, Podoba JE Ramman-Haddad L. Environmental risk factors for delirium in hospitalized older people. J Am Geriatr Soc 2001;49:1327-34

3 US Department of Health and Human Services. 2004 CMS statistics. Washington, DC: Centers for Medicare and Medicaid Services, 2004:34. (CMS Publication No 03445.)

4 Siddiqi N, Horne AO, House AO, Holmes JD. Occurrence and outcome of delirium in medical in-patients; a systematic literature review. Age Ageing 2006;35:350-64.

5 Adamis D, Martin FC, Treloar A, Macdonald AJD. Capacity, consent, and selection bias in a study of delirium. J Med Ethics 2005;31:137-43.

6 McCusker J, Cole M, Dendukuri N, Han L, Bedzile E. The course of delirium in older medical inpatients: a prospective study. I Gen Intern Med 2003;18:696-704.

7 McAvay GJ, van Ness PH, Bogardus ST, Zhany H, Leslie DL, Leo-Summers LS, et al. Older adults discharged from hospital with delirium: one year outcomes. J Am Geriatr Soc 2006; 54:1245-50

8 American Psychiatric Association. Diagnostic and statistical manual of mental disorders. 4th ed. Washington, DC: APA 1994.

9 O'Keeffe ST, Lavan JN. Clinical significance of delirium subtypes in older people. Age Ageing 1999;28:115-9.

10 Practice guideline for the treatment of patients with delirium American Psychiatric Association, 1999. (www.psych.org/ psych_pract/treatg/pg/Practice\%20Guidelines8904/ Delirium.pdf)

11 The prevention, diagnosis and management of delirium in older people. National guidelines. 2006. www.rcplondon.ac.uk/pubs/ books/pdmd/index.asp

12 O'Keeffe ST, Mulkerna EC, Nayeem K, Verughese M, Pillay I. Use of serial mini-mental state examinations to diagnose and monitor delirium in elderly hospital patients. J Am Geriatr Soc 2005;53:867-70.

13 Inouye SK, van Dyck CH, Alessi CA, Balkin S, Siegal AP, Horwitz RI. Clarifying confusion: the confusion assessment method. Am Coll Phys 1990;113:941-8.

14 Inouye SK. The confusion assessment method (CAM): training manual and coding guide 2003. Boston: Yale University School of Medicine, 2003.

15 Fick DM, Agostini JV, Inouye SK. Delirium superimposed on dementia: a systematic review. J Am Geriatr Soc 2002;50:1723-32.

16 Inouye SK, Charpentier PA. Precipitating risk factors for delirium in hospitalised elderly persons: predictive model and inter-relationship with baseline vulnerability. JAMA 1996; 275:852-7.

17 Alagiakrishnan K, Wiens CA. An approach to drug induced delirium in the elderly. Postgrad Med / 2004;80:388-93.

18 Ancelin ML, Artero S, Portet F, Dupuy AM, Touchon J, Richie K. Non-degenerative mild cognitive impairment in elderly people and use of anticholinergic drugs: longitudinal cohort study. BMJ 2006;332:455-9.

19 Siddiqi N, Stockdale R, Holmes J, Britton AM. Interventions for preventing delirium in hospitalised patients. Cochrane Database Systematic Review (in press).

20 Inouye SK, Bogardus ST, Charpentier PA, Leo-Summers L, Acampora D, Holford TR, et al. A multicomponent intervention to prevent delirium in hospitalized older patients. $N$ Engl J Med 1999;340:669-76.

21 Marcantonio ER, Flacker JM, Wright J, Resnick NM. Reducing delirium after hip fracture: a randomized trial. J Am Geriatr Soc 2001;49:516-22.

22 Inouye SK, Bogardus T, Williams CS, Leo-Summers L, Agostini JV. The role of adherence on the effectiveness of nonpharmacologic interventions: evidence from the delirium prevention trial. Arch Intern Med 2003;163:958-64.

23 Inouye SK, Bogardus ST, Baker DI, Leo-Summers L, Cooney LM The hospital elder life program: a model of care to prevent cognitive and functional decline in hospitalized older patients. J Am Geriatr Soc 2000;48:1697-706.

24 Caplan GA, Coconis J, Board N, Sayers A, Woods J. Does home treatment affect delirium? A randomised controlled trial of rehabilitation of elderly and care at home or usual treatment (the REACH-OUT trial). Age Ageing 2006;35:53-60.

25 Cole MG, McCusker J, Bellavance F, Primeau FJ, Bailey RF, Bonnycastle MJ, et al. Systematic detection and multidisciplinary care of delirium in older medical inpatients: a randomized trial. CMAI 2002;167:753-9. 